\title{
EXCITATION OF DEEP DEFECTS BY INTENSE TERAHERTZ RADIATION
}

\author{
S.D. GanicheV \\ Institut für Experimentelle und Angewandte Physik, Universität Regensburg \\ 93040 Regensburg, Germany \\ and \\ A.F. Ioffe Physicotechnical Institute, Russian Academy of the Sciences \\ 194021 St. Petersburg, Russia
}

\begin{abstract}
An analysis is done of the ionization of deep impurity centers by high-intensity terahertz radiation, with photon energies tens of times lower than the impurity ionization energy. Under these conditions, ionization can be described as direct tunneling and phonon-assisted tunneling in which carrier emission is accompanied by defect tunneling in configuration space and electron tunneling in the electric field of the radiation. Within a broad range of intensity, frequency, and temperature, the terahertz electric field of the radiation acts like a static field. For very high frequencies and low temperatures an enhancement of tunneling as compared to static fields was observed. The transition between the quasi-static and the high frequency regime is determined by the tunneling time. For the case of deep impurities this is the time of redistribution of the defect vibrational system which depends strongly on temperature and the impurity structure.
\end{abstract}

PACS numbers: 71.55.-i, 72.20.Ht, 72.40.+w, 72.30.+q

\section{Introduction}

The effect of high-frequency coherent radiation on tunneling in semiconductor superlattices and nanostructures has recently attracted considerable a.tention. The superposition of a static electric field and an alternating field causes a wealth of new phenomena as a result of photon assisted tunneling [1-3]. In all these cases tunneling is accomplished by a static electric field and the radiation influences the barrier penetration probability. An intense radiation field, however, can in fact generate both the tunneling barrier and initiate tunneling. Such a tunneling process has been observed for deep impurities in semiconductors [4]: In contrast to the tunneling ionization of atoms, where only electron tunneling takes place [5], phonon-assisted tunneling ionization of impurities in solids is accomplished by two simultaneous tunneling processes, electron tunneling and the redistribution of the vibrational system by defect tunneling. With increasing electric field strength, the direct carrier tunneling from the ground state into continuum, without participation of phonons, becomes dominant. 


\section{Theoretical consideration}

In most cases deep impurities have one bound state which phenomenologically can be approximated by a potential well. The emission and capture of electrons or holes by deep impurities in semiconductors can be considered in the adiabatic approximation. Due to electron-phonon interaction the system consisting of local impurity vibrations and the electron is characterized by two adiabatic potentials $U_{1}(x)$ and $U_{2}(x)$ as a function of a configuration coordinate $x$ (see insets in Fig. 3). Following the Huang-Rhys model these adiabatic potentials correspond to the electron bound to the impurity and to the electron detached from the impurity with zero kinetic energy, respectively. The energy separation between the minima of $U_{1}$ and $U_{2}$ is the thermal ionization energy of the electron $\varepsilon_{\mathrm{T}}$. Thermal emission of carriers from the bound state into the continuum is accomplished by thermal excitation of the system in the adiabatic bound state potential $U_{1}$ to a vibrational energy $\mathcal{E}_{1}$ and tunneling of the impurity configuration from this state into the ionized configuration $U_{2}$ with energy $\mathcal{E}_{2}$ (Fig. 1) [6-9].

a)

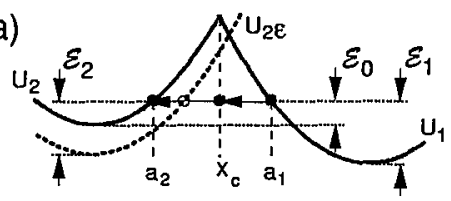

b)

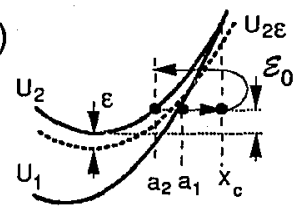

Fig. 1. Tunneling trajectories for the ionization of autolocalized (a) and substitutional (b) deep impurities.

In the presence of an electric field the electron can be emitted at a negative kinetic energy $-\varepsilon$ due to tunneling through the triangular potential barrier formed by the potential well and the electric field (see inset in Fig. 4). Hence, the adiabatic potential of the ionized configuration $U_{2}$ is shifted to a lower energy $U_{2 \varepsilon} \equiv U_{2}-\varepsilon$ (Figs. 1,3). Thus electron emission in an electric field is achieved by two tunneling processes, electron tunneling with probability $P_{\mathrm{e}}$ and tunneling of the defect from the adiabatic potential $U_{1}(x)$ to potential $U_{2 \varepsilon}$ with the probability $P_{\mathrm{d}}$.

The ionization probability may be written as

$$
e(E)=\iint P_{\mathrm{e}} P_{\mathrm{d}} \exp \left(-\mathcal{E}_{1} / k_{\mathrm{B}} T\right) \mathrm{d} \varepsilon \mathrm{d} \mathcal{E}_{1} .
$$

The Boltzmann factor takes into account the thermal excitation of the system in the adiabatic potential $U_{1}$.

In the semi-classical approximation, neglecting pre-exponential factors, the defect tunneling probability is given by $[7,9]$

$$
\begin{aligned}
& P_{\mathrm{d}} \propto \exp \left[-2\left(S_{2} \mp S_{1}\right)\right], \\
& S_{i}\left(\mathcal{E}_{i}\right)=\frac{\sqrt{2 M}}{\hbar} \int_{a_{i}}^{x_{\mathrm{c}}} \mathrm{d} x \sqrt{U_{i}(x)-\mathcal{E}_{i}}, \quad i=1,2,
\end{aligned}
$$

where $M$ is a mass corresponding to the mode of impurity vibration, $a_{i}$ and $x_{\mathrm{c}}$ are shown in Fig. 1, and the minus and plus signs are related to the particular impurity configuration shown in Fig. $1 \mathrm{a}$ and b, respectively. 
Electron tunneling in an alternating electric field $E=E_{0} \cos (\omega t)$ has been treated theoretically by Keldysh [5] (see also [10]). The tunneling probability is given by

$$
P_{\mathrm{e}} \propto \exp \left[-2 S_{\mathrm{e}}(\varepsilon)\right], \quad S_{\mathrm{e}}(\varepsilon)=-\frac{\varepsilon}{\hbar} \int_{0}^{\tau_{\mathrm{e}}} \frac{1}{\gamma^{2}} \sinh ^{2}(\omega \tau) \mathrm{d} \tau+\frac{\varepsilon \tau_{\mathrm{e}}}{\hbar} .
$$

Here $\gamma \equiv \sqrt{2 m^{*} \varepsilon} \omega / e E$ and $\sinh \left(\omega \tau_{\mathrm{e}}\right)=\gamma ; m^{*}$ and $e$ are the electron effective mass and charge, respectively, and $\tau_{\mathrm{e}}=\hbar \partial S_{\mathrm{e}} / \partial \varepsilon$ has the meaning of an electron tunneling time $[11,12]$.

The integral Eq. (1) was calculated using the saddle point method. In the case of weak electric fields, i.e., as long as the saddle point energy $\varepsilon \ll \varepsilon_{\mathrm{T}}$, the exponent in the index can be developed into a power series of $\varepsilon$. Taken into account that $\mathcal{E}_{2}=\mathcal{E}_{1}-\left(\varepsilon_{\mathrm{T}}-\varepsilon\right)$ we obtain

$$
\left.S_{2} \mp S_{1} \approx\left(S_{2} \mp S_{1}\right)\right|_{\varepsilon=0}-\frac{\varepsilon \tau_{2}}{\hbar}, \quad \text { with } \quad \tau_{2}=\left.\frac{\hbar \partial\left|S_{2}\right|}{\partial \mathcal{E}_{2}}\right|_{\varepsilon=0} .
$$

Then the saddle point condition gives

$$
\tau_{2}=\tau_{\mathrm{e}}, \quad \tau_{2}=\frac{\hbar}{2 k_{\mathrm{B}} T} \pm \tau_{1}
$$

where $\tau_{1}=\hbar \partial\left|S_{1}\right| /\left.\partial \mathcal{E}_{1}\right|_{\varepsilon=0}$. The first term in Eq. (5) states that the electron tunneling time $\tau_{\mathrm{e}}$ is equal to the defect tunneling time $\tau_{2}$ for tunneling under the potential $U_{2}$.

Finally we obtain for the ionization probability

$$
\begin{aligned}
& e(E) \propto \exp \left(\frac{E^{2}}{E_{\mathrm{c}}^{* 2}}\right)=\exp \left[\frac{E^{2} e^{2}\left(\tau_{2}^{*}\right)^{3}}{3 m^{*} \hbar}\right], \\
& \left(\tau_{2}^{*}\right)^{3}=\frac{3}{4 \omega^{3}}\left[\sinh \left(2 \omega \tau_{2}\right)-2 \omega \tau_{2}\right] .
\end{aligned}
$$

This result shows that $\left(\tau_{2}^{*}\right)^{3}$ increases exponentially as a function of $\omega \tau_{2}$. As the ionization probability itself depends exponentially on the third power of $\tau_{2}^{*}$ the tunneling ionization is drastically enhanced with rising $\omega \tau_{2}$. This can be achieved not only by increasing the radiation frequency $\omega$ but also by lowering the temperature (Eq. (5)). In the limit $\omega \tau_{2} \ll 1$ the time constant $\tau_{2}^{*}$ approaches the tunneling time $\tau_{2}$ giving the result of the static field regime obtained in [13].

\section{Samples and experimental technique}

The tunneling ionization of deep impurities by high-intensity far-infrared (FIR) radiation with photon energies much smaller than the thermal impurity ionization energy $\varepsilon_{\mathrm{T}}$ was studied for different types of deep impurities: substitutional impurities with weak electron-phonon coupling (Ge:Au, Ge:Hg, Ge:Cu, Ge:Zn, $\mathrm{Si}: \mathrm{Au}, \mathrm{GaP}: T e$ with acceptor concentrations in the range $\left.10^{14}-10^{15} \mathrm{~cm}^{-3}\right)$ and autolocalized $\mathrm{DX}^{-}$centers with strong electron phonon coupling $\left(\mathrm{Al}_{x} \mathrm{Ga}_{1-x} \mathrm{As}: \mathrm{Te}\right.$, $\mathrm{Al}_{x} \mathrm{Ga}_{1-x} \mathrm{Sb}$ :Te with $x=0.35$ and 0.5 ). In the investigated temperature range from $4.2 \mathrm{~K}$ to $80 \mathrm{~K}$, for autolocalized impurities up to $150 \mathrm{~K}$, practically all impurities are occupied in thermal equilibrium. 
The change of the ionization probability in the electric field which is proportional to the change in the free carrier concentration can be detected as photoconductive signal under FIR-laser illumination. The samples were placed in a temperature-controlled optical cryostat. Penetration of light in the medium IR range into the cryostat was prevented by the use of crystalline quartz filters, and in the visible, with a $1 \mathrm{~mm}$ thick black-polyethylene filter. Measurements were carried out using a standard $50 \Omega$ load resistor circuit, taking care that the bias voltage across the sample was substantially below the threshold of electric breakdown, which for bulk samples is at about $5 \mathrm{~V} / \mathrm{cm}$.

The radiation sources used was a pulsed far-infrared molecular laser optically pumped by a TEA $\mathrm{CO}_{2}$ laser. The $\mathrm{CO}_{2}$ laser was used for optical pumping because of its wavelength range $9.2-10.6 \mu \mathrm{m}$, which includes strong vibrational-rotational absorption lines of many molecules. Commercial TEA $\mathrm{CO}_{2}$ lasers (URENKO-204) offer high stability and high power to assemble such a system. The characteristics of strong single lines in the terahertz range from $3.78 \mathrm{THz}$ to $68 \mathrm{THz}$, the corresponding gain media, and lines of the TEA $\mathrm{CO}_{2}$ laser used for pumping are listed in Table [14]. The radiation pulse length varies for different lines from 10 to $100 \mathrm{~ns}$. The radiation is focused to a spot of about $1 \mathrm{~mm}^{2}$, with the maximum intensity reaching as high as $5 \mathrm{MW} / \mathrm{cm}^{2}$ corresponding to electric fields up to about $50 \mathrm{kV} / \mathrm{cm}$. The intensity, pulse shape, and the spatial distribution of the laser radiation were measured with fast room temperature photodetectors based on the photon drag effect [15], intraband $\mu$-photoconductivity [16] and the Spirikon pyroelectric camera, respectively.

TABLE

Characteristics of the lasing lines used in this work.

\begin{tabular}{c|c|c|c}
\hline \hline $\begin{array}{c}\text { Wavelength } \\
{[\mu \mathrm{m}]}\end{array}$ & $\begin{array}{c}\text { Line of } \mathrm{CO}_{2} \\
\text { pump laser }\end{array}$ & $\begin{array}{c}\text { Maximum FIR intensity } \\
{\left[\mathrm{kW} / \mathrm{cm}^{2}\right]}\end{array}$ & Medium \\
\hline 35 & $10 \mathrm{P}(24)$ & 300 & $\mathrm{NH}_{3}$ \\
66 & $9 \mathrm{P}(32)$ & 100 & $\mathrm{D}_{2} \mathrm{O}$ \\
76 & $10 \mathrm{P}(26)$ & 4000 & $\mathrm{NH}_{3}$ \\
90.5 & $9 \mathrm{R}(16)$ & 5000 & $\mathrm{NH}_{3}$ \\
148 & $9 \mathrm{P}(36)$ & 4500 & $\mathrm{NH}_{3}$ \\
152 & $10 \mathrm{P}(32)$ & 3000 & $\mathrm{NH}_{3}$ \\
250 & $9 \mathrm{R}(26)$ & 400 & $\mathrm{CH}_{3} \mathrm{~F}$ \\
256 & $10 \mathrm{R}(14)$ & 500 & $\mathrm{NH}_{3}$ \\
280 & $10 \mathrm{R}(8)$ & 1000 & $\mathrm{NH}_{3}$ \\
385 & $9 \mathrm{R}(22)$ & 5 & $\mathrm{D}_{2} \mathrm{O}$ \\
496 & $9 \mathrm{R}(20)$ & 10 & $\mathrm{CH}_{3} \mathrm{~F}$
\end{tabular}




\section{Experimental results and analysis}

Ionization of deep impurities by far-infrared radiation was observed for all samples in the whole frequency range investigated. A photoconductive signal increasing nonlinearly with incident power $\left(I \leq 5 \mathrm{MW} / \mathrm{cm}^{2}, E \leq 5 \times 10^{4} \mathrm{~V} / \mathrm{cm}\right)$ was observed in spite of the fact that the photon energies were much smaller than the binding energy of the impurities. Ionization can be attributed to phonon-assisted and direct tunneling over a wide range of temperature, frequency, and electric field strength [14].

At not too low temperatures and not too high frequencies the tunneling probability is independent on frequency and exponentially increases with the square of the electric field strength* (see Eq. (6)). This is illustrated in Fig. 2a where experimental results obtained with AlGaAs:Te at $T=150 \mathrm{~K}$ are shown. In this quasi-static regime the electron tunnels at the momentary magnitude of the electric field in a time shorter than the period of oscillation and thus the electric field

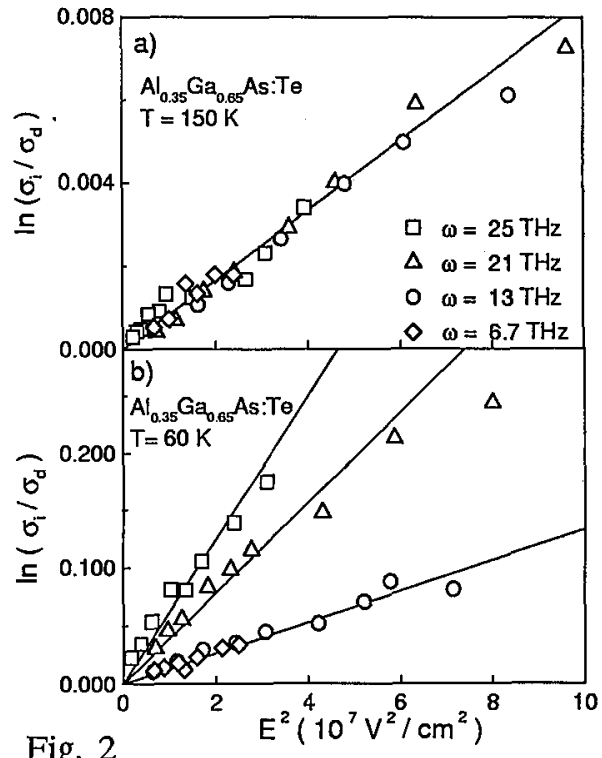

Fig. 2

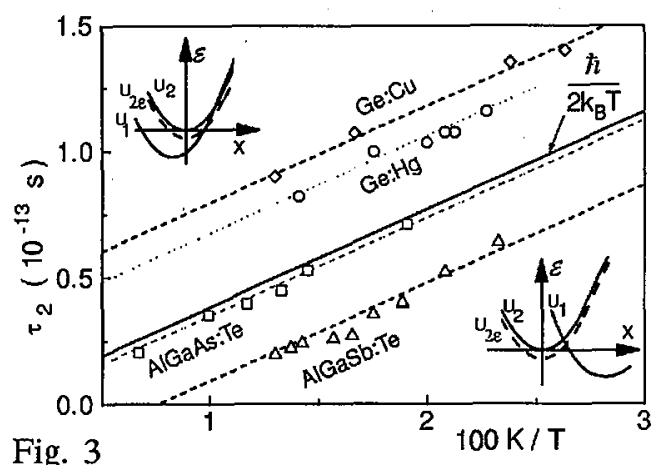

Fig. 3

Fig. 2. The ionization probability (given by the ratio of the conductivity under illumination and in the dark $\sigma_{\mathrm{i}} / \sigma_{\mathrm{d}}$ ) of $\mathrm{Al}_{0.35} \mathrm{Ga}_{0.65} \mathrm{As}$ : Te as a function of the square of the electric field of the radiation.

Fig. 3. Tunneling times $\tau_{2}$ as a function of $1 / T$ for different samples. The full line shows $\hbar / 2 k_{\mathrm{B}} T$, the broken lines are plotted according to Eq. (5). Insets: adiabatic potentials for substitutional impurities (top left) and autolocalized impurities (bottom right).

* In the case of charged impurities (all substitutional impurities investigated) a deviation from the field dependence $e(E) \propto \exp \left(E^{2} / E_{c}^{* 2}\right)$ can be seen in relatively low field strength (up to $1 \mathrm{kV} / \mathrm{cm}$ ) where the defects are thermally ionized through the Poole-Frenkel effect and the ionization probability is proportional to $e(E) \propto \exp \left(\sqrt{Z e^{3} E / \kappa} / k_{\mathrm{B}} T\right)[14,17-19]$. 
acts like a static field. Such a behavior has been observed for all materials at suffciently high temperatures. In this quasi-static regime $[14,19,20]$ the characteristic field, which can be determined experimentally, is given by $E_{\mathrm{c}}^{* 2}=3 m^{*} \hbar /\left(\tau_{2}^{* 3} e^{2}\right)$ with $\tau_{2}^{*}=\tau_{2}$. Thus the investigation of field dependence of ionization probability allows to determine the defect tunneling time $\tau_{2}$. Figure 3 presents the temperature dependence of the tunneling time $\tau_{2}$ obtained for various samples. For the purpose of comparison, $\hbar / 2 k_{\mathrm{B}} T$ is also plotted in Fig. 3. As can be seen, $\tau_{2}$ is larger than $\hbar / 2 k_{\mathrm{B}} T$ for substitutional impurities and smaller than $\hbar / 2 k_{\mathrm{B}} T$ for the DX-centers. Thus the tunneling time reflects the structure of the potential barriers which is systematically distinct for both potential configurations discussed here. The phonon-assisted ionization of deep impurities in semiconductors by contactless application of short pulses of terahertz radiation has been proposed as a new method for the characterization of defects [14, 20-22]. The field dependence of the signal allows one to determine the defect tunneling times, the Huang-Rhys parameter and the basic structure of the adiabatic defect potentials. The Poole-Frenkel effect, which can be observed for charged impurities only (see footnote), can be used to determine the defect charge state.

This frequency independent tunneling is limited to frequencies $\omega$ with $\omega \tau_{2}<1$ (Eq. (7)). The enhancement of tunneling at frequencies higher than the reciprocal tunneling time has been anticipated in a number of theoretical papers $[5,10,23-25]$ but has been demonstrated experimentally only recently [26]. In contrast to static electric fields where the electron tunnels at a fixed energy, in alternating fields the energy of the electron is not conserved during tunneling (see the inset in Fig. 4). In this case the electron can absorb energy from the field, which leads to a sharp increase in the tunneling probability with increasing frequency. This effect was observed for various impurities in different semiconductors and is demonstrated for AlGaAs:Te in Fig. 2b. For the case of $\omega \tau_{2} \geq 1$ the

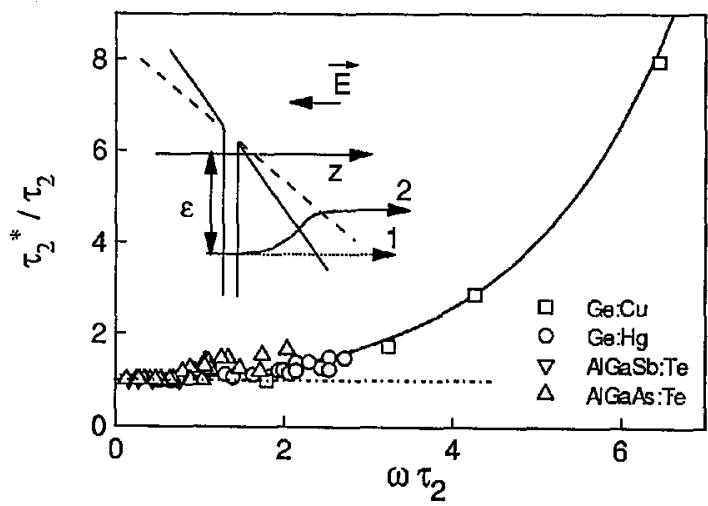

Fig. 4. Ratio $\tau_{2}^{*} / \tau_{2}$ as a function of $\omega \tau_{2}$. The line shows the dependence according to Eq. (7). Inset: electron tunneling trajectory: (1) in a static electric field, and (2) in an alternating field. 
ionization probability still depends exponentially on the square of the electric field strength but the characteristic field becomes frequency dependent and ionization is drastically enhanced with rising frequency.

In Fig. 4 the ratio $\tau_{2}^{*} / \tau_{2}$ calculated after Eq. (7) is plotted as a function of $\omega \tau_{2}$ and compared to experimental results obtained from measured values of $E_{\mathrm{c}}^{*}$ for various frequencies, temperatures, and materials. The tunneling times $\tau_{2}$ were determined from frequency independent values of $E_{c}^{*}$. The experimental results shown in Fig. 4 are grouped according to the materials. For each material the variation of the value of $\omega \tau_{2}$ was obtained by applying different radiation frequencies in the range from $6.7 \mathrm{THz}$ to $25 \mathrm{THz}$ and different temperatures between $20 \mathrm{~K}$ and $150 \mathrm{~K}$. It should be pointed out that the theory leading to good agreement with experiment does not contain any fitting parameters.

Further decrease in the temperature leads to a much stronger frequency dependence of the ionization probability. Figure 5 shows experimental results for $\mathrm{Ge}: \mathrm{Cu}$ at $T=4.2 \mathrm{~K}$ in the frequency range between 3.4 and $25 \mathrm{THz}$. In order to display in one figure the total set of data covering eight order of magnitude in the square of the electric field, $\log \left(E^{2}\right)$ was plotted on the abscissa. To make an easy comparison to the $\exp \left(E^{2} / E_{\mathrm{c}}^{2}\right)$ dependence of $\sigma_{\mathrm{i}} / \sigma_{\mathrm{d}}$ possible, a $\log -\log$ presentation was used for the ordinate.

At $4.2 \mathrm{~K}$ the condition $\omega \tau_{2} \gg 1$ is valid for the whole frequency range investigated here. The measurements of Fig. 5 show that at liquid helium temperature in the limit of $\omega \tau_{2} \gg 1$ a drastic frequency dependence is observed. For a given constant signal a change of six orders of magnitude of electric fields squared needs

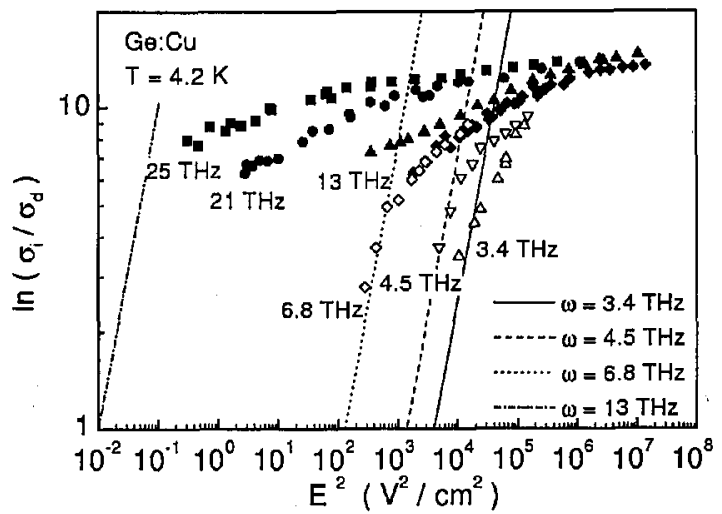

Fig. 5. The ionization probability (given by the ratio of the conductivity under illumination and in the dark $\sigma_{\mathrm{i}} / \sigma_{\mathrm{d}}$ ) of $\mathrm{Ge}: \mathrm{Cu}$ at $4.2 \mathrm{~K}$ as a function of the square of the electric field of the radiation. Solid symbols are obtained with molecular optically pumped FIR laser with frequencies $-25 \mathrm{THz}, \bullet-21 \mathrm{THz}$, full $\Delta-13 \mathrm{THz}$, full $\diamond-6.8 \mathrm{THz}$, respectively. Open symbols are obtained with Santa Barbara Free-Electron-Laser with frequencies $\diamond-6.8 \mathrm{THz}, \nabla-4.5 \mathrm{THz}, \triangle-3.4 \mathrm{THz}$, respectively [26]. Straight lines show the dependence according to Eqs. (6), (7) for four lowest frequencies used in experiment. 
only a seven times change in frequency. Note that this large variation in the field strength yielding the same signal is valid only for the case of relatively low fields. In Fig. 5 in addition the result of calculations for phonon-assisted tunneling with $\tau_{2}$ determined at higher temperatures is plotted. It is seen that for the three lowest frequencies the experimental points at low field strength are reasonably well described by the calculated emission probabilities of phonon assisted tunneling after Eqs. (6) and (7). At the same time for higher frequencies the calculated field dependence of phonon-assisted tunneling does not fit the experimental results in the whole range of electric fields where a signal could be detected. Furthemore, at higher field strength the field dependence of the emission probability is much weaker and, as is seen in Fig. 5, the frequency dependence practically disappears.

This complex dependence of ionization probability on field strength and radiation frequency is a result of the transition from phonon-assisted tunneling at low field strength to direct tunneling without involving phonons at high fields. The emission probability for phonon-assisted tunneling as a function of the electric field strength given by Eq. (6) was obtained in the limit that corrections to thermal emission resulting from electron tunneling are small, i.e., the energy of electron tunneling $\varepsilon$ is much smaller than the defect tunneling energy $\mathcal{E}_{0}$ and the energy of thermal ionization $\varepsilon_{\mathbf{T}}$. In the opposite limit, $\varepsilon>\varepsilon_{\mathbf{T}}, \mathcal{E}_{0}$, direct carrier tunneling from the ground state into the continuum, without participation of phonons, becomes dominant. Direct electron tunneling occurs at the crossing of the $U_{2 \varepsilon}$ and $U_{1}$ potential curves, where an electronic transition is possible without any change in the configuration coordinate. This effect, leading to weaker growth of the ionization probability in comparison to the field dependence of phonon-assisted tunneling, extrapolated to higher fields, determines the ionization process at very high fields $[14,19,27]$.

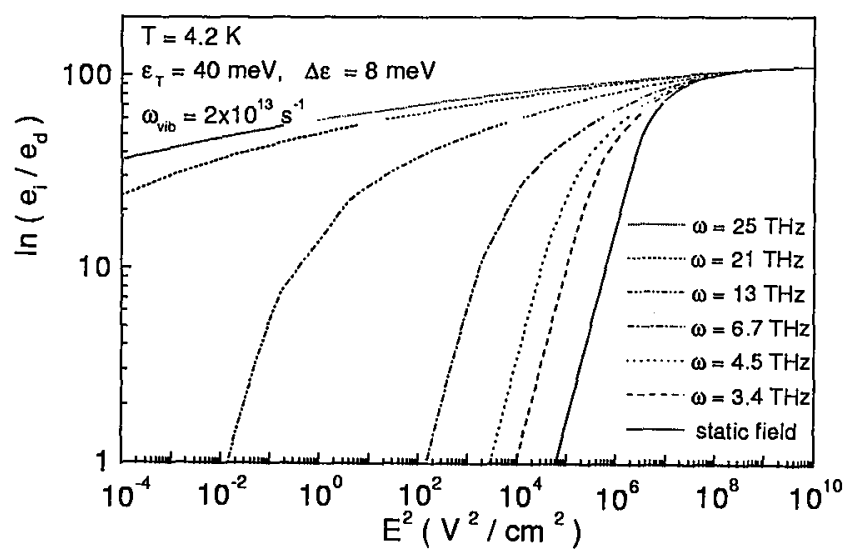

Fig. 6. Field dependence of ionization probability calculated after Eqs. (1)-(3) for all values of the energy of electron tunneling $\varepsilon$ for $\mathrm{Ge}: \mathrm{Cu}$ at $4.2 \mathrm{~K}$ and frequencies used in the experiments taking into account phonon-assisted and direct tunneling but ignoring the Coulomb interaction. 


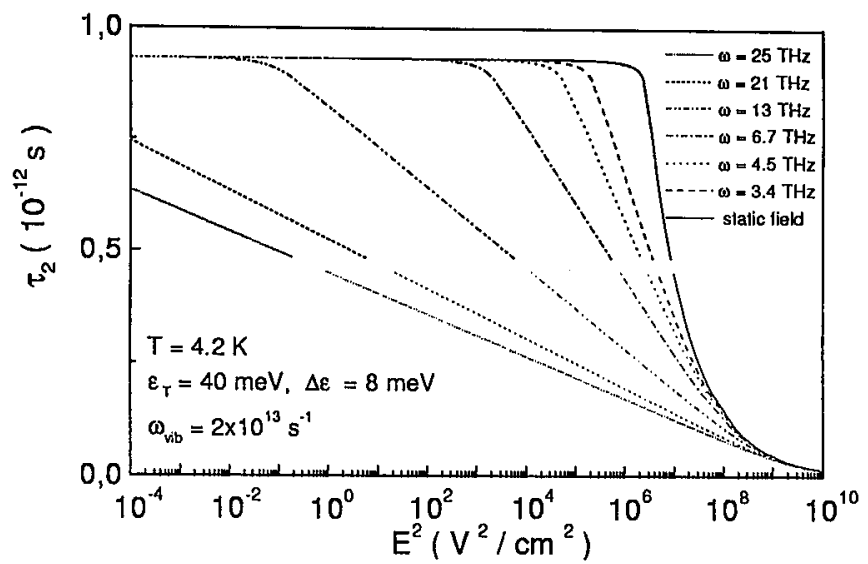

Fig. 7. The field dependence of tunneling time $\tau_{2}$ calculated after Eqs. (1)-(3) for all values of the energy of electron tunneling $\varepsilon$ for $\mathrm{Ge:Cu}$ at $4.2 \mathrm{~K}$ and frequencies used in the experiments taking into account phonon-assisted and direct tunneling but ignoring the Coulomb interaction.

Figure 6 shows the result of calculations after Eqs. (1)-(3) for all values of the electron tunneling energy $\varepsilon$ for $\mathrm{Ge}: \mathrm{Cu}$ at $4.2 \mathrm{~K}$ and frequencies used in the experiments taking into account both processes but ignoring the Coulomb interaction. It is seen that the theory describes well the experimentally observed features of the field and frequency dependence of tunneling ionization. The disappearance of the frequency dependence of the ionization probability at high field strengths is caused by a decrease in the tunneling time $\tau_{2}$ with rising electric field strength (Fig. 7) finally giving $\omega \tau_{2}$ smaller than unity. This is due to the fact that at high field strength, defect tunneling occurs at energies much smaller than the energy of phonon-assisted tunneling which changes the tunneling trajectory. To achieve the quantitative agreement one needs to improve the theory by taking into account the charge of the impurities.

Finally, our measurements were carried out with deep impurities in semiconductors, however, because tunneling is crucial in numerous processes in physics, chemistry, and biology we expect that an enhancement of tunneling by contactless application of coherent radiation will have significant consequences.

\section{Acknowledgments}

Financial support by the DFG and the RFFI are gratefully acknowledged. The author would like to thank W. Prettl, I.N. Yassievich, V.I. Perel, and E. Ziemann for active help and discussion.

\section{References}

[1] P.S.S. Guimaraes, B.J. Keay, J.P. Kaminski, S.J. Allen, Jr., P.F. Hopkins, A.C. Gossard, L.T. Florez, J.P. Harbinson, Phys. Rev. Lett. 70, 3792 (1993).

[2] B.J. Keay, S.J. Allen, Jr., J. Gallan, J.P. Kaminski, K.L. Campman, A.C. Gossard, U. Bhattacharya, J.W. Rodwell, Phys. Rev. Lett. 75, 4098 (1995). 
[3] C.J.G.M. Langerak, B.N. Murdin, B.E. Cole, J.M. Chamberlain, M. Henini, M. Pate, G. Hill, Appl. Phys. Lett. 67, 3453 (1995).

[4] S.D. Ganichev, W. Prettl, P.G. Huggard, Phys. Rev. Lett. 71, 3882 (1993).

[5] L.V. Keldysh, Sov. Phys.-JETP 20, 1307 (1965).

[6] S. Makram-Ebeid, M. Lannoo, Phys, Rev. B 25, 6406 (1982).

[7] T. Markvart, J. Phys. C 17, 6303 (1984).

[8] P.T. Landsberg, Recombination in Semiconductors, Cambridge University Press, New York 1991.

[9] V.N. Abakumov, V.I. Perel, I.N. Yassievich, in: Nonradiative Recombination in Semiconductors, Eds. V.M. Agranovich, A.A. Maradudin, Modern Problems in Condensed Matter Sciences, Vol. 33, North-Holland, Amsterdam 1991.

[10] L.D. Landau, E.M. Lifshitz, Quantum Mechanics, Pergamon, Oxford 1977, p. 287.

[11] M. Büttiker, R. Landauer, Phys. Rev. Lett. 49, 1739 (1982).

[12] R. Landauer, Th. Martin, Rev. Mod. Phys. 66, 217 (1994).

[13] V. Karpus, V.I. Perel, Sov. Phys.-JETP 64, 1376 (1986).

[14] S.D. Ganichev, W. Prettl, I.N. Yassievich, review in Phys. Solid State 39, 1703 (1997).

[15] S.D. Ganichev, Ya.V. Terent'ev, I.D. YaroshetskiY, Sov. Tech. Phys. Lett. 11, 20 (1985).

[16] S.D. Ganichev, S.A. Emel'yanov, A.G. Pakhomov, Ya.V. Terent'ev, I.D. YaroshetskiY, Sov. Tech. Phys. Lett. 11, 377 (1985).

[17] S.D. Ganichev, J. Diener, I.N. Yassievich, W. Prettl, Europhys. Lett. 29, 315 (1995).

[18] S.D. Ganichev, E. Ziemann, W. Prettl, A. Istratov, E.R. Weber, to be published, MRS Symp. Proc., San Francisco 1999.

[19] S.D. Ganichev, I.N. Yassievich, W. Prettl, Semicond. Sci. Technol. 11, 679 (1996).

[20] S.D. Ganichev, J. Diener, I.N. Yassievich, W. Prettl, B.K. Meyer, K.W. Benz, Phys. Rev. Lett. 75, 1590 (1995).

[21] E. Ziemann, S.D. Ganichev, I.N. Yassievich, K. Schmalz, W. Prettl, Mater. Res. Soc. Symp. Proc. 510, 595 (1998).

[22] S.D. Ganichev, W. Raab, E. Zepezauer, W. Prettl, I. Yassievich, Phys. Rev. B 55, 9243 (1997).

[23] Yu.A. Bychkov, A.M. Dykhne, Sov. Phys.-JETP 31, 928 (1970).

[24] B.I. Ivlev, V.I. Mel'nikov, Phys. Rev. Lett. 55, 1614 (1985).

[25] M.V. Ammosov, N.B. Delone, V.P. Krainov, Sov. Phys.-JETP 64, 1191 (1986).

[26] S.D. Ganichev, E. Ziemann, Th. Gleim, W. Prettl, I.N. Yassievich, V.I. Perel, I. Wilke, E.E. Haller, Phys. Rev. Lett. 80, 2409 (1998).

[27] S.D. Ganichev, J. Diener, W. Prettl, Solid State Commun. 92, 883 (1994). 